\title{
Das geplante Bundesgesetz über polizeiliche Massnahmen zur Bekämpfung von Terrorismus (PMT) - Verfassungsgrundlage und
} Verfahrensrecht

Terrorismus stellt auch für die Schweiz eine ernstzunehmende Bedrohung dar. Dieser soll mit einem neuen Bundesgesetz über polizeiliche Massnahmen zur Bekämpfung des Terrorismus (PMT) begegnet werden. Der vorliegende Beitrag argumentiert, dass dem Bund für den Erlass der Massnahmen zur Verhinderung terroristischer Aktivitäten, welche den Kern des PMT bilden, eine hinreichende verfassungsrechtliche Grundlage fehlt. Hinzu kommt, dass die geplanten Massnahmen einen weiten und wenig präzisen Anwendungsbereich haben, aber gleichzeitig präventive und erhebliche Grundrechtseingriffe ermöglichen würden. Diese Belastung der Betroffenen wird auch nicht verfahrensrechtlich kompensiert. Vielmehr beherrscht eindeutig das Bundesamt für Polizei das Verfahren. Eine gerichtliche Prüfung der Massnahmen findet grundsätzlich nur im Nachhinein statt und wenn der angebliche terroristische Gefährder überhaupt ein Rechtsmittel ergreift.

I. Einleitung

II. Bundesgesetz über polizeiliche Massnahmen zur Bekämpfung von Terrorismus (PMT) $\ldots \ldots \ldots \ldots$

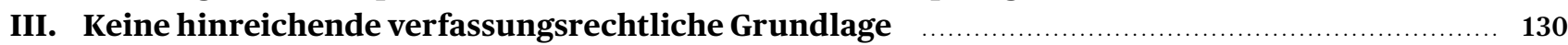

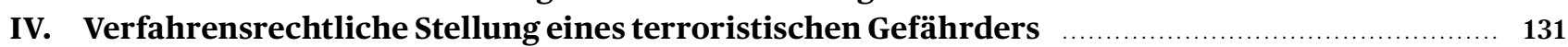

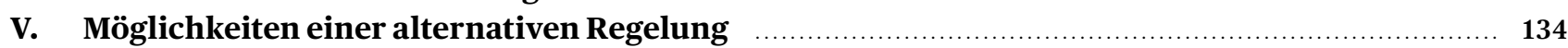

VI. Fazit ……

Zitiervorschlag:

PATRICE MARTIN ZUMSTEG, Das geplante Bundesgesetz über polizeiliche Massnahmen zur Bekämpfung von Terrorismus (PMT) - Verfassungsgrundlage und Verfahrensrecht, sui generis 2021, S. 125

Dr. iur. Patrice Martin Zumsteg, wissenschaftlicher Mitarbeiter und Dozent für Staats- und Verwaltungsrecht an der ZHAW Zürcher Hochschule für Angewandte Wissenschaften, Winterthur, Rechtsanwalt bei Abegg Anwälte und Konsulenten, Zürich, (zumg@zhaw.ch). Der Autor dankt Prof. Dr. iur. Anna Coninx für ihr Manuskript zum Thema und ein ausführliches Gespräch.

URL: sui-generis.ch/176

DOI: https://doi.org/10.21257/sg.176

Dieses Werk ist lizenziert unter einer Creative Commons Namensnennung - Weitergabe unter gleichen Bedingungen 4.0 International Lizenz. 


\section{Einleitung}

1 Nach Einschätzung des Nachrichtendienstes des Bundes (NDB) ist die Terrorbedrohung in der Schweiz seit 2015 erhöht, wobei die grösste Gefahr für unser Land von islamistischen Extremisten ausgeht. ${ }^{1}$ Die Bekämpfung des Terrorismus soll vor allem auch durch Prävention erfolgen. ${ }^{2}$ Dies zeigt sich im Nationalen Aktionsplan zur Verhinderung und Bekämpfung von Radikalisierung und gewalttätigem Extremismus (NAP), ${ }^{3}$ mit welchem Präventions- und Integrationsmassnahmen beschlossen wurden. Im Weiteren wurde eine Ergänzung des StGB 4 angestossen. ${ }^{5}$ Das dritte grosse Projekt des NAP auf Bundesebene ist der Erlass des hier zu betrachtenden Bundesgesetzes über polizeiliche Massnahmen zur Bekämpfung von Terrorismus (PMT), 6 gegen welches das Referendum zustande gekommen ist. ${ }^{7}$

2 Nachfolgend wird ein Überblick über das gesamte PMT gegeben, das ein Mantelerlass ist. ${ }^{8}$ Sodann werden die darin vorgesehenen Massnahmen zur Bekämpfung von Terrorismus dargestellt. ${ }^{9}$ Es wird untersucht, ob diese auf einer hinreichenden verfassungsrechtlichen Grundlage beruhen und welche verfahrensrechtlichen Absicherungen zugunsten der von den Massnahmen Betroffenen bestehen. Im Hinblick darauf werden die geplanten Massnahmen schliesslich bewertet und eigene Revisionsvorschläge gemacht.

1 Nachrichtendienst des Bundes, Sicherheit Schweiz 2020, S.35ff.

2 Botschaft vom 18. September 2015 zur Strategie der Schweiz zur Terrorismusbekämpfung (BBl 20157487), S. 7492 f.

3 Sicherheitsverbund Schweiz, Nationaler Aktionsplan zur Verhinderung und Bekämpfung von Radikalisierung und gewalttätigem Ex tremismus, vom 4. Dezember 2017.

4 Schweizerisches Strafgesetzbuch vom 21. Dezember 1937 (StGB SR311.0).

5 Einen Überblick gibt MARCO TRAGLIA, Evolution de la législation suisse contre le terrorisme en bref, Jusletter vom 5 . November 2018 N $5 \mathrm{ff}$. Vgl. auch SVEN ZIMMERLIN, Das Bundesgesetz über polizeiliche Massnahmen zur Bekämpfung von Terrorismus, Sicherheit \& Recht 2020, S. $185 \mathrm{ff}$.

6 Bundesgesetz vom 25. September 2020 über polizeiliche Massnah men zur Bekämpfung von Terrorismus (BBl 2020 7741).

7 Referendum gegen das Bundesgesetz vom 25. September 2020 über polizeiliche Massnahmen zur Bekämpfung von Terrorismus (PMT) (BBl 2021461)

8 Botschaft vom 22. Mai 2019 zum Bundesgesetz über polizeiliche Massnahmen zur Bekämpfung von Terrorismus (BBl 2019 4751), S. 4847.

9 Diese bilden den Kern des PMT, vgl. Botschaft PMT (Fn. 8), S. 4847; ZIMMERLIN (Fn. 5), S.189.

\section{Bundesgesetz über polizeiliche Massnahmen zur Bekämpfung von Terrorismus (PMT)}

\section{Grundzüge der Vorlage}

Das PMT enthält zur Hauptsache eine Teilrevision des 3 BWIS ${ }^{10}$. Daneben sind mit dem PMT auch Anpassungen in weiteren Bundesgesetzen vorgesehen, insbesondere im Migrationsrecht, im StGB, im ZentG ${ }^{11}$ und im BPI'12. Die sich im Zusammenhang mit diesen Teilrevisionen stellenden Fragen bilden nicht Gegenstand dieses Beitrags. ${ }^{13}$ Im Folgenden wird auf die Massnahmen fokussiert, welche in das BWIS eingefügt werden sollen.

Der Titel des 5. Abschnitts des BWIS bezeichnet sie als 4 «Massnahmen zur Verhinderung terroristischer Aktivitäten». Es handelt sich um eine Melde- und Gesprächsteilnahmepflicht (nArt. 23k BWIS), ein Kontakt- und ein Rayonverbot (nArt. $23 l$ und 23m BWIS), ein Ausreiseverbot (nArt. 23n BWIS) sowie die Eingrenzung auf eine Liegenschaft («Hausarrest», nArt. 230 BWIS). Zur Begründung sowie zur Durchführung der Massnahmen können besonders schützenswerte Personendaten bearbeitet und zwischen den Behörden ausgetauscht werden (nArt. 23h BWIS). Zum Vollzug der Massnahmen nach nArt. 23l-23o BWIS ist zudem eine elektronische Überwachung oder Lokalisierung des terroristischen $\mathrm{Ge}$ fährders über Mobilfunk möglich (nArt. 23q BWIS).

\section{Teilrevision des BWIS}

\section{a) Materielles}

Zweck der Massnahmen nach nArt. 23k-23q BWIS (BWIS- 5 Massnahmen) ist es, radikalisierte Personen mit polizeilichem Zwang an bestimmten Tätigkeiten zu hindern. Eine fundamentalistische oder terroristische Gesinnung allein soll als Auslöser nicht hinreichend sein. Erst wenn darüber hinaus eine terroristische Aktivität droht, sollen die Behörden handeln können. ${ }^{14}$ Mit Ausnahme der Eingrenzung auf eine Liegenschaft handelt es sich um Massnahmen, welche auch bereits in anderen Erlassen

10 Bundesgesetz über Massnahmen zur Wahrung der inneren Sicherheit vom 21. März 1997 (BWIS; SR 120).

11 Bundesgesetz über die kriminalpolizeilichen Zentralstellen des Bundes und gemeinsame Zentren für Polizei- und Zollzusammenarbeit mit anderen Staaten vom 7. Oktober 1994 (ZentG; SR 360).

12 Bundesgesetz über die polizeilichen Informationssysteme des Bundes vom 13.Juni 2008 (BPI; SR 361).

13 Eine Kommentierung der einzelnen Artikel findet sich in der Botschaft PMT (Fn. 8), S. 4804 ff.

14 Botschaft PMT (Fn. 8), S. 4756 und 4785; ZIMMERLIN (Fn. 5), S. 193. 
verankert sind ${ }^{15}$ - so etwa in der StPO16, im sogenannten Hooligan-Konkordat' ${ }^{17}$ oder in kantonalen Gesetzen wie dem PolG/ZH18 und dem GSG/ZH'19. Das neue Instrument der Eingrenzung i.S.v. nArt. 230 BWIS kann erst angeordnet werden, wenn ein Verstoss gegen eine Massnahme gestützt auf die nArt. 23k-23n BWIS vorliegt (nArt. 230 Abs. 1 lit. b BWIS). ${ }^{20}$

6 Sämtliche Massnahmen knüpfen am neuen Begriff der terroristischen Gefährderin an. ${ }^{21}$ Als solche gilt «eine Person, wenn aufgrund konkreter und aktueller Anhaltspunkte davon ausgegangen werden muss», dass sie eine «terroristische Aktivität» ausüben wird (nArt. 23e Abs. 1 BWIS). Das umfasst alle Bestrebungen, welche die staatliche Ordnung beeinflussen oder verändern sollen und die durch «Begehung oder Androhung von schweren Straftaten oder mit der Verbreitung von Furcht und Schrecken verwirklicht oder begünstigt werden sollen» (nArt. 23e Abs. 2 BWIS). ${ }^{22}$ Als Gefährderin kann grundsätzlich qualifiziert werden, wer das 12. Altersjahr vollendet hat (nArt. $24 f$ Abs. 1 BWIS). ${ }^{23}$

7 Noch vor Inkrafttreten dieser Bestimmungen ist internationale Kritik am weiten Anwendungsbereich laut geworden. Die Menschenrechtskommissarin des Europarats hielt fest, dass die Definition des terroristischen Gefährders ihrer Auffassung nach nicht hinreichend präzise und klar sei, um das Risiko einer willkürlichen Anwendung der Bestimmungen auszuschliessen. Zudem sei ungewiss, ob ausserhalb des Systems des Jugendstrafrechts genügend Rücksicht auf die besondere Schutzbedürftigkeit von Minderjährigen genommen werde. ${ }^{24}$ In

15 Botschaft PMT(Fn. 8), S. 4766; JÜRG MARCEL TIEFENTHAL, Kantonales Polizeirecht der Schweiz, Zürich 2018, \$\$8, 12, 16, 23, 27; ZIMMERLIN (Fn. 5), S.190, m.w.H.

16 Schweizerische Strafprozessordnung vom 5. Oktober 2007 (StPO SR312.0).

17 Konkordat über Massnahmen gegen Gewalt anlässlich von Sportveranstaltungen vom 15 . November 2007, Text abrufbar über Gesetz über den Beitritt zum Konkordat über Massnahmen gegen Gewalt anlässlich von Sportveranstaltungen des Kantons Zürich vom 18. Mai 2009 (LS 551.19).

18 Polizeigesetz des Kantons Zürich vom 23. April 2007 (PolG/ZH; LS 550.1).

19 Gewaltschutzgesetz des Kantons Zürich vom 19.Juni 2006 (GSG/ZH; LS 351).

20 Vgl. Botschaft PMT (Fn. 8), S. 4796.

21 KASTRIOT LUBISHTANI / HADRIEN MONOD, Mesures policières de lutte contre le terrorisme, Analyse critique du projet de loi fédérale Sicherheit \& Recht 2020, S. 20.

22 «Terroristische Aktivität» ist analog zu Art.19 Abs. 2 lit. a Bundesgesetz über den Nachrichtendienst vom 25. September 2015 (NDG; SR121) zu verstehen, vgl. Botschaft PMT (Fn. 8), S. 4783.

23 Eine Erhöhung der Altersgrenze wurde im Parlament abgelehnt. Bundesrätin Keller-Sutter wies in der Beratung auf dieselbe Grenze in Art. 11 Hooligan-Konkordat und auf die Grenze für die Strafmündigkeit (10. Altersjahr) hin, vgl. AB2020 S $90 \mathrm{ff.}$

24 La Commissaire aux Droits de l'Homme, CommHR/DM/sf 014-2020, 7. Mai 2020. Weitere Hinweise zur Frage des Mindestalters bei ZIMMERLIN (Fn. 5), S. 190. ähnlicher Weise hielten fünf Sonderberichterstatter der UNO dafür, dass die begrifflichen Definitionen sehr weit seien und potenziell auch journalistische und andere legitime Aktivitäten der Zivilgesellschaft erfassen könnten. ${ }^{25}$

Tatsächlich ist festzustellen, dass die in nArt.23e BWIS 8 eingeführten Begriffe wesentlich auf die Zukunft abstellen, die naturgemäss ungewiss ist, womit auch die entsprechende Norm stark an Vorhersehbarkeit verliert. ${ }^{26}$ Die bundesrätliche Botschaft bejaht die erforderlichen aktuellen und konkreten Anhaltspunkte schon dann, wenn sich jemand allmählich aus seinem bisherigen sozialen Umfeld zurückzieht und neue Kontakte in ein Umfeld knüpft, in dem terroristische Gewalt gerechtfertigt oder verherrlicht wird. Als Beispiel werden auch Aktivitäten in den sozialen Medien angeführt, wie «das Weiterverbreiten terroristischer Inhalte etwa durch das «Verlinken» oder durch das «Befürworten», das Setzen von sogenannten Likes».27 Das Gesetz soll also zeitlich sehr weit vor einer unmittelbaren Gefahr oder einem tatsächlich ausgeführten terroristischen Anschlag zur Anwendung kommen. ${ }^{28}$ Diese Unbestimmtheit und mangelhafte Vorhersehbarkeit steht im Widerspruch zu den Anforderungen an die Normdichte bei wichtigen Bestimmungen. ${ }^{29}$

Gleichzeitig können die Massnahmen für mehrere Mo- 9 nate angeordnet werden, was sie deutlich von polizeilichen Zwangsmassnahmen unterscheidet, wie sie die Kantone kennen. ${ }^{30}$ Die erste Anordnung ist auf sechs Monate begrenzt und kann einmalig um maximal sechs Monate verlängert werden (nArt.23g Abs.1 BWIS). Sie kann allerdings erneut angeordnet werden, wenn neue und konkrete Anhaltspunkte für eine terroristische Aktivität vorliegen (nArt. 23 Abs. 2 BWIS). Eine Ausnahme

25 FIONNUALA Ní AOLÁIN / AGNES CALLAMARD / DAVID KAYE / AHMED SHAHEED / NILS MELZER, Commentaires à propos du projet de loi intitulé «Loi fédérale sur les mesures policières de lutte contre le terrorisme», OL CHE 1/2020.

26 Gl.M. ANNA CONINX, Neue Terrorismusbekämpfung in der SchweizGrundlagen und Kritik, unveröffentlichtes Manuskript 2021, S. 10; Botschaft PMT (Fn. 8), S. 4784, räumt immerhin prognostische «Unsicherheiten» ein.

27 Botschaft PMT (Fn. 8), S. 4760 (Hervorhebung im Original).

28 Gl.M. CONINX (Fn. 26), S.11; vermittelnd ZIMMERLIN (Fn. 5), S. 190 und $192 \mathrm{f}$.

29 BGE143 II162 E. 3.2.1; BGE140 I 381 E. 4.4; Urteil des EGMR [Plenum] 6538/74 vom 26. April 1979 (The Sunday Times gegen das Vereinigte Königreich Nr.1), Ziff. 49; Urteil des EGMR 11801/85 vom 24. April 1990 (Kruslin gegen Frankreich), Ziff. $32 \mathrm{ff}$.

30 So etwa \$27 Abs. 2 und \$33 PolG/ZH (polizeilicher Gewahrsam sowie Wegweisung und Fernhaltung grundsätzlich nur für 24 Stunden). Nach dem Hooligan-Konkordat ist der Polizeigewahrsam ebenfalls auf 24 Stunden beschränkt (Art. 8 Abs. 2); für Wegweisungen und Rayonverbote muss ein strafbares Verhalten tatsächlich festgestellt worden sein (Art. 4 Abs. 1 und Art. 6 Abs.1). Vgl. auch DANIEL MOECKLI / RAPHAEL KELLER, Wegweisungen und Rayonverbote - ein Überblick, Sicherheit \& Recht 2012, S. 234. 
gilt für die Eingrenzung auf eine Liegenschaft, die auf insgesamt maximal neun Monate beschränkt ist (nArt. 230 Abs. 5 BWIS). ${ }^{31}$ Die Massnahmen sollen vor, während und nach einem allfälligen Strafverfahren ausgesprochen werden können. ${ }^{32}$

\section{b) Verwaltungsrechtlicher Charakter der Massnahmen}

10 Die BWIS-Massnahmen sind eingriffsintensiv. ${ }^{33}$ Mit ihnen kann über mehrere Monate hinweg insbesondere in die persönliche Freiheit, die Meinungsäusserungsfreiheit, die Versammlungs- und Vereinigungsfreiheit sowie in die Glaubens- und Gewissensfreiheit eingegriffen werden - Garantien, welche nicht nur in der BV ${ }^{34}$, sondern auch in der EMRK ${ }^{35}$, dem UNO-Pakt II ${ }^{36}$ und weiteren völkerrechtlichen Verträgen der Schweiz verankert sind. ${ }^{37}$ Eine ausführliche Grundrechtsprüfung unterbleibt hier mit Rücksicht auf den Umfang des Beitrags.

11 Das BWIS selbst charakterisiert die Massnahmen als vorbeugend-polizeilich (Art. 2 Abs. 2 nlit. dbis BWIS), was auch die Absicht des Gesetzgebers war. ${ }^{38}$ Sie sind also nicht repressiv-strafrechtlicher Natur und haben keine punitive Funktion, sondern dienen der Gefahrenabwehr und haben präventiven Charakter. ${ }^{39}$ Das gilt auch für die Eingrenzung, welche sich freiheitsentziehend auswirkt und damit eine gewisse Nähe zu straf- und strafprozessrechtlichen Massnahmen hat. ${ }^{40}$ Das Bundesgericht sprach der Meldeauflage, dem Rayonverbot und dem Polizeigewahrsam nach dem Hooligan-Konkordat eine «öffentlich-rechtliche, verwaltungsrechtliche Natur» zu. ${ }^{41} \mathrm{Die}$ se Qualifikation lässt sich m.E. auf die Massnahmen des BWIS übertragen. ${ }^{42}$ Neben dem Konkordat dienen auch

31 Eine erneute Anordnung ist danach ausgeschlossen, vgl. Botschaft PMT (Fn. 8), S. 4797.

32 Botschaft PMT (Fn. 8), S. 4760.

33 So auch die Botschaft PMT (Fn. 8), S. 4785; LUBISHTANI/MONOD (Fn. 21), S. 23.

34 Bundesverfassung der Schweizerischen Eidgenossenschaft vom 18. April 1999 (BV; SR 101).

35 Konvention zum Schutze der Menschenrechte und Grundfreiheiten vom 4. November 1950 (EMRK; SR 0.101).

36 Internationaler Pakt über bürgerliche und politische Rechte vom 16. Dezember 1966 (UNO-Pakt II; SR 0.103.2).

37 Botschaft PMT (Fn. 8), S. 4759; LUBISHTANI/MONOD (Fn. 21), S. 23; ZIMMERLIN (Fn. 5), S.194f.

38 Botschaft PMT (Fn. 8), S. 4757.

39 Zu den Begriffen: Josianne MAGnIN, Die Polizei: Aufgaben, rechts staatliche Grenzen und Haftung, Diss. Luzern 2016, Zürich 2017, S.10 ff.; MARKUS MOHLER, Grundzüge des Polizeirechts in der Schweiz, Basel 2012, N806 ff.

40 Ausführlich ANDREAS DONATSCH, Rechtsgutachten Umgang mitge fährlichen Personen, Mögliche gesetzgeberische Lösungen auf Stufe Bund und Kantone, Zürich 2019, passim und insb. N23 ff. Vgl. auch LUBISHTANI/MONOD (Fn. 21), S. 23.

41 BGE137 I 31 E. 4.3 und 4.4. Vgl. auch Urteil des Bundesverwaltungsgerichts C-2397/2014 vom 19. Februar 2015 E.3.5.

42 Kritisch, aber im Ergebnis gleich: LUBISHTANI/MONOD (Fn. 21), S. 22. die meisten anderen der erwähnten Erlasse primär der Gefahrenabwehr. ${ }^{43}$

Bei den BWIS-Massnahmen handelt es sich damit um 12 präventiv-polizeiliche Massnahmen. ${ }^{44}$ Es wird nachfolgend geprüft, ob dem Bund dafür eine hinreichende Rechtsetzungskompetenz zukommt. ${ }^{45}$

\section{c) Verfahren der Anordnung im Allgemeinen}

Die BWIS-Massnahmen treten neben die sozialen, inte- 13 grativen oder therapeutischen Massnahmen, welche insbesondere durch die Kantone angeordnet werden (nArt. $23 f$ Abs. 1 lit. a und b sowie Abs. 2 BWIS). ${ }^{46}$ Ebenso sollen die neuen BWIS-Massnahmen auch neben Zwangsmassnahmen, die sich auf die StPO stützen, treten können (vgl. nArt. $23 f$ Abs. 1 lit. c BWIS). ${ }^{47}$ Dieses Nebeneinander macht den Austausch und die Abstimmung zwischen zahlreichen Behörden notwendig. ${ }^{48}$ Grundlage für die Bearbeitung von besonders schützenswerten Personendaten (etwa über religiöse und weltanschauliche Ansichten) von terroristischen Gefährdern ist nArt. $23 h$ Abs. 1 BWIS. ${ }^{49}$ Die Daten können zwischen Polizei- und Strafverfolgungsbehörden, den Kindes- und Erwachsenenschutzbehörden, Schulen und Bildungsbehörden sowie Integrationsfachstellen, Einwohner-, Migrations-, Jugend- und Sozialämtern ausgetauscht werden, soweit das für die Anordnung und den Vollzug der Massnahmen erforderlich ist (nArt. 23h Abs. 2 BWIS). 50

Verfügt werden die Massnahmen gestützt auf nArt. 23j 14 Abs. 1 Satz1 BWIS durch das Bundesamt für Polizei (fedpol). Aufgrund des Subsidiaritätsprinzips ist dazu grundsätzlich ein Antrag der kantonalen oder kommunalen Behörden erforderlich. Geht der Antrag vom NDB aus, ist der betroffene Kanton anzuhören (nArt. $23 i$ Abs. 1 und nArt. 23j Abs. 1 Satz 3 BWIS). ${ }^{51}$ Im Antrag ist darzulegen, dass die gesetzlichen Voraussetzungen erfüllt sind

43 Vgl. Art.1 Hooligan-Konkordat («vorbeugende polizeiliche Massnahmen»), \$3 PolG/ZH («Aufrechterhaltung der öffentlichen Sicherheit und Ordnung») und $\$ 1$ GSG/ZH («vorbeugende Massnahmen»).

44 Gl.M. MARKUS MOHLER, Dem PMT-Gesetz fehlt die Verfassungsgrundlage, sui generis 2021, S. 61 ff., N2; ZIMMERLIN (Fn. 5), S.189; vgl. auch LUBISHTANI/MONOD (Fn. 21), S. 22. Statt "präventiv-repres siv» wird auch «Sicherheitspolizei-Kriminalpolizei» verwendet, vgl. PIERRE TSCHANNEN, Normenarchitektur des Bundes, in: Kiener/ Bühler/Schindler (Hrsg.), Schweizerisches Bundesverwaltungsrecht Band III, Sicherheits- und Ordnungsrecht des Bundes, Teil 2 Besonderer Teil, Basel 2018, N1 ff. und $19 \mathrm{ff}$., BWIS als ein Haupterlass im Bereich der Sicherheitspolizei.

45 Vgl. unten III.

46 Botschaft PMT (Fn. 8), S. 4785f.; LUBISHTANi/MONOD (Fn. 21), S. 21.

47 Botschaft PMT (Fn. 8), S. $4786 \mathrm{f}$.

48 Botschaft PMT (Fn. 8), S. 4789; ZIMMERLIN (Fn. 5), S.196f.

49 Und in engem Umfang auch von Drittpersonen (zum Beispiel bei einem Kontaktverbot), vgl. Botschaft PMT (Fn. 8), S. 4788.

50 Botschaft PMT (Fn. 8), S. 4789.

51 Botschaft PMT (Fn. 8), S. 4789 ff. und 4767. 
(nArt. 23i Abs. 2 BWIS). 52 Dem fedpol sind sämtliche relevanten Unterlagen zu übermitteln. ${ }^{53}$ Der Vollzug und die Kontrolle der Massnahmen bleibt in der Verantwortung der Kantone (nArt. 23r BWIS), die sich auf ihre jeweiligen Polizeigesetze stützen. ${ }^{54}$

Im Einvernehmen mit dem betroffenen Kanton respektive der betroffenen Gemeinde kann fedpol eine Massnahme sistieren, wenn wichtige Gründe vorliegen (nArt. $23 j$ Abs. 3 BWIS). ${ }^{55}$ Sie ist von Amtes wegen ganz aufzuheben, wenn deren Voraussetzungen nicht mehr erfüllt sind (nArt. 23 A Abs. 3 BWIS). Schliesslich kann die Betroffene bei fedpol jederzeit ein Gesuch um Aufhebung der Massnahmen stellen (nArt. $23 f$ Abs. 4 BWIS) und damit eine Überprüfung derselben erwirken. ${ }^{56}$

\section{d) Verfahren der Anordnung beim «Hausarrest»}

Besondere Verfahrensvorschriften bestehen für die Eingrenzung auf eine Liegenschaft i.S.v. nArt. 230 BWIS. Zwar hat fedpol auch diesbezüglich Verfügungsbefugnis (vgl. nArt. 23j Abs.1 BWIS) und tritt die Massnahme umgehend in Kraft. ${ }^{57}$ Allerdings muss es eine entsprechende Anordnung dem Zwangsmassnahmengericht des Kantons Bern «zur Prüfung der Rechtmässigkeit und Angemessenheit» unterbreiten. Dieses entscheidet innert höchstens 48Stunden nach Eingang des entsprechenden Antrags (nArt. 23p Abs. 1 BWIS). Letzterer muss Angaben zu Art und Umfang der Bedrohung, zur Verletzung bisheriger Anordnungen (nArt. 230 Abs. 1 lit.b BWIS) und zu sämtlichen für die Beurteilung der Verhältnismässigkeit relevanten Umstände enthalten. ${ }^{58}$

17 Eine Verlängerung der Eingrenzung muss fedpol dem Gericht spätestens vier Tage vor Ablauf beantragen. Das Gericht hat allerdings nicht zwingend innert dieser Frist und auch nicht innert 48 Stunden zu entscheiden. Vielmehr hat es die Möglichkeit, die Fortdauer der Eingrenzung als vorsorgliche Massnahme für die Dauer des Verfahrens anzuordnen (nArt. $23 p$ Abs. 2 BWIS). 59

18 Der Betroffene kann fedpol ein schriftlich begründetes Gesuch um Aufhebung der Massnahme einreichen. Wenn fedpol das Gesuch ablehnt, leitet es dieses und eine begründete Stellungnahme innert drei Tagen weiter an das

\footnotetext{
52 Insbesondere ist darzulegen, welche Massnahmen bereits ergriffen wurden und inwiefern diese nicht genügen, vgl. Botschaft PMT (Fn. 8), S. 4790.

53 Botschaft PMT (Fn. 8), S. 4790.

54 Botschaft PMT (Fn. 8), S.4801. Die Vollzugsbehörden können sich nicht zusätzlich auf das BWIS berufen, vgl. ZIMMERLIN (Fn. 5), S.196.

55 Aber wohl nicht auf Antrag der Betroffenen, vgl. Botschaft PMT (Fn. 8), S. 4791.

56 Botschaft PMT (Fn. 8), S. 4787.

57 Botschaft PMT (Fn. 8), S. 4797; ZIMMERLiN (Fn. 5), S. 195.

58 Botschaft PMT (Fn. 8), S. 4797.

59 Botschaft PMT (Fn. 8), S. 4797 f.
}

Zwangsmassnahmengericht zum Entscheid innert weiterer fünf Tage (nArt. 23p Abs. 4 BWIS). Bei Gutheissung wird die Eingrenzung unverzüglich beendet. Ebenso wenn die Voraussetzungen für die Anordnung wegfallen oder das Gericht die ursprüngliche Anordnung oder eine später beantragte Verlängerung verweigert (nArt. 23p Abs. 5 BWIS).

\section{e) Rechtsschutz}

Verfügungen von fedpol als auch Entscheide des Zwangs- 19 massnahmengerichts unterliegen der Beschwerde an das Bundesverwaltungsgericht (nArt. $24 g$ Abs. 1 BWIS). Das Rechtsmittelverfahren richtet sich nach den allgemeinen Bestimmungen der Bundesrechtspflege. ${ }^{60} \mathrm{Ne}-$ ben dem Verfügungsadressaten (Art. 48 Abs.1 VwVG61) sind auch die antragstellende kantonale oder kommunale Behörde gegen Verfügungen von fedpol und dieses gegen Entscheide des Zwangsmassnahmengerichts zur Beschwerde berechtigt (nArt. $24 \mathrm{~g}$ Abs. 2 BWIS). ${ }^{62}$ Es können sämtliche Beschwerdegründe i.S.v. Art. 49 VwVG vorgebracht werden. ${ }^{63}$

Die Beschwerde hat grundsätzlich keine aufschiebende 20 Wirkung, weil der Gesetzgeber annimmt, dass den Gefährdungen unverzüglich mit den BWIS-Massnahmen begegnet werden muss. ${ }^{64}$ Sie sind demnach sofort vollstreckbar (Art. 39 lit. b VwVG). ${ }^{65}$ Die aufschiebende Wirkung kann erteilt werden, wenn der Zweck der Massnahme dadurch nicht gefährdet wird (nArt. $24 g$ Abs. 3 BWIS).

Das Urteil des Bundesverwaltungsgerichts unterliegt 21 der Beschwerde in öffentlich-rechtlichen Angelegenheiten an das Bundesgericht (Art. 82 lit. a und Art. 86 Abs. 1 lit. a BGG66).67

60 Botschaft PMT (Fn. 8), S. 4802; ZIMMERLIN (Fn. 5), S. 197.

61 Bundesgesetz über das Verwaltungsverfahren vom 20. Dezember 1968 (VwVG; SR 172.021).

62 Die kantonale oder kommunale Behörde kann einen Entscheid des Zwangsmassnahmengerichts nicht weiterziehen, ZIMMERLIN (Fn. 5), S. 195 .

63 ZIMMERLIN (Fn. 5), S. 197.

64 Botschaft PMT (Fn. 8), S. 4803.

65 ZIMMERLIN (Fn. 5), S. 197. Allgemein zur Vollstreckung: REGINA KIENER / BERNHARD RÜTSCHE / MATHIAS KUHN, Öffentliches Verfahrensrecht, 2. Aufl., Zürich 2015, N852ff.

66 Bundesgesetz über das Bundesgericht vom 17.Juni 2005 (BGG; SR173.110).

67 ZIMMERLIN (Fn. 5), S.197. Zur Beschwerde in öffentlich-rechtlichen Angelegenheiten: RENÉ RHINOW / HEINRICH KOLLER / CHRISTINA KISS / DANIELA THURNHERR / DENISE BRÜHL-MOSER, Öffentliches Prozessrecht, Grundlagen und Bundesrechtspflege, 3. Aufl., Basel 2014, N1853 ff.; KIENER/RÜTSCHE/KUHN (Fn. 65), N1388ff., je m.w.H. 


\section{Keine hinreichende verfassungs- rechtliche Grundlage}

PMT-Vorlage stützt sich gemäss ihrem Ingress au Art. 54 Abs. 1 (Auswärtige Angelegenheiten), Art. 57 Abs. 2 (Sicherheit), Art.123 Abs.1 (Strafrecht) und Art. 173 Abs. 2 BV (Weitere Aufgaben und Befugnisse der Bundesversammlung). Der Bundesrat hält dazu fest, dass die effiziente Bekämpfung terroristischer Bedrohungen «ein einheitliches und koordiniertes Vorgehen» voraussetze. Der Einbezug des Bundes stelle sicher, dass die Fäden auf einer Ebene zusammenlaufen würden. Dem Bund komme eine Rechtsetzungsbefugnis für den Erlass des PMT zu. ${ }^{68}$ Das ist für die Teilrevision des BWIS näher zu prüfen.

Soweit es den Bereich der äusseren Sicherheit i.S.v. Art. 54 Abs. 1 BV betrifft, ist dem Bundesrat zuzustimmen, dass in dieser Hinsicht eine umfassende Bundeskompetenz besteht. ${ }^{69}$ Traditionell wird die äussere von der inneren Sicherheit nach der Gefahrenquelle differenziert, die im Inland (z.B. Kriminalität) oder im Ausland (z.B. militärische Bedrohungen) liegen kann. Zu Recht wird darauf hingewiesen, dass diese Unterscheidung mit neuen Bedrohungsformen - wie dem hier relevanten Terrorismus - zunehmend schwierig wird. ${ }^{70}$ Allerdings ist daran festzuhalten, weil dies für die Zuordnung der Kompetenzen nach geltendem Verfassungsrecht ausschlaggebend ist. ${ }^{71}$ Der Bund kann aus seiner Zuständigkeit für die «auswärtigen Angelegenheiten» (Art. 54 Abs. 1 BV) ableiten, dass er für die internationale Sicherheitskooperation sowie weitere Felder mit Auslandsbezug zuständig ist. ${ }^{72}$ Zum Erlass von polizeilichen Massnahmen im Innern der Schweiz kann sich der Bund aber nicht auf diese Kompetenz stützen. ${ }^{73}$

\footnotetext{
68 Botschaft PMT (Fn. 8), S. 4763f. und 4845f.

69 GIOvANNI BIAGgINI, BV Kommentar, 2. Aufl., Zürich 2017, Art. 57 N 5 (zit. BIAGGINI, OFK BV); RAINER J. SCHWEIZER / MARKUS MOHLER, in: Ehrenzeller/Schindler/Schweizer/Vallender (Hrsg.), Die schweizerische Bundesverfassung, St. Galler Kommentar, 3. Aufl., Zürich 2014, Vorbem. zur Sicherheitsverfassung N24 (zit. SGK BV-BEARBEITERIN).

70 OLIVER DIGGELMANN / TILMANN ALTWICKER, in: Waldmann/Belser/Epiney (Hrsg.), Basler Kommentar Bundesverfassung, Basel 2015, Art. 57 N 20 (zit. BSK BV-BEARBEITERIN); SGK BV-SCHWEIZER/ MOHLER, Vorbem. zur Sicherheitsverfassung N11.

71 TSCHANNEN, Normenarchitektur (Fn. 44), N 5; Bericht des Bundesrates vom 2. März 2012 in Erfüllung des Postulats Malama 10.3045 vom 3. März 2010, Innere Sicherheit. Klärung der Kompetenzen (BBl 20124459), S. 4478.

72 DANIEL MOECKLI, Sicherheitsverfassung, in: Diggelmann / Hertig Randall / Schindler (Hrsg.), Verfassungsrecht der Schweiz, Band 3, Zürich 2020, N11f.; SGK BV-SCHWEIZER/MOHLER, Vorbem. zur Sicherheitsverfassung N27ff.

73 Gl.M. LUBiSHTANi/MONOD (Fn. 21), S. 25 f.; SGK BV-SCHWEIZER/ MOHLER, Vorbem. zur Sicherheitsverfassung N33; teilweise a.A. TSCHANNEN, Normenarchitektur (Fn. 44), N14.
}

Zutreffend ist, dass die Sorge für die Sicherheit von Land 24 und Bevölkerung eine staatliche Kernaufgabe ist. ${ }^{74}$ Allerdings liegt die Polizeihoheit - welche hier angesprochen ist - bei den Kantonen. ${ }^{75}$ Sie sind für die Wahrung der inneren Sicherheit auf ihrem Gebiet zuständig, sofern sich aus der BV keine Zuständigkeit des Bundes ergibt. ${ }^{76}$ Aus Art. 57 Abs. 1 BV kann der Bund keine solche Zuständigkeit ableiten. ${ }^{77}$

Art. 57 Abs. 2 BV, der im Ingress zwar angerufen, in der 25 Botschaft aber nur am Rande erwähnt wird, ${ }^{78}$ begründet nach herrschender Lehre ebenfalls keine Bundeskompetenz, was sich insbesondere aus dem Wortlaut und dem Grundsatz der Subsidiarität (Art. 3, 5a sowie Art. 42 und 47 BV) ergibt. ${ }^{79}$ Vorliegend ist bemerkenswert, dass im Gesetzgebungsprozess wiederholt auf das Hooligan-Konkordat verwiesen, ${ }^{80}$ aber nicht erklärt wurde, weshalb statt dem PMT nicht ein Konkordat zwischen den Kantonen angestrebt wurde. Ein solches könnte die fraglichen Massnahmen enthalten und sich auf eine klare Rechtsetzungskompetenz abstützen. ${ }^{81}$

Der angerufene Art. 123 Abs.1 BV, der die Gesetzgebung 26 auf dem Gebiet des Straf- und Strafprozessrechts zur Bundessache erklärt, ist eine hinreichende Grundlage für die vorgesehene Teilrevision des StGB. ${ }^{22}$ Nicht von dieser Bestimmung gedeckt sind jedoch polizeiliche Massnahmen, wie sie neu im BWIS aufgenommen werden sollen. ${ }^{83}$

Ebenso wenig begründet schliesslich Art. 173 Abs. 2 BV 27 Kompetenzen zugunsten des Bundes - vielmehr muss sich eine solche nach den üblichen Regeln der Verfassungsauslegung ergeben. ${ }^{84}$ Dabei ist anerkannt, dass neben expliziten auch inhärente Bundeskompetenzen

74 BIAGgini, OFK BV, Vorbem. Art. 57-61 N1; MOECKLI (Fn.72), N8; PIERRE TSCHANNEN, Staatsrecht der Schweizerischen Eidgenossenschaft, 4. Aufl., Bern 2016, §1 N10.

75 Bericht Malama (Fn. 71), S. 4479 f.; BIAGgini, OFK BV, Art. 57 N 5; MOHLER, Grundzüge (Fn.39), N57.

76 Bericht Malama (Fn. 71), S. 4478f.; MOECKLI (Fn. 72), N8f., m.w.H.

77 BIAGGINI, OFK BV, Art. 57 N2; BSK BV-DIGGELMANN/ALTWICKER, Art. 57 N23; MOECKLI (Fn.72), N8f.

78 Botschaft PMT (Fn. 8), S. 4847.

79 BIAGGINI, OFK BV, Art. 57 N10; BSK BV-DIGGELMANN/ALTWICKER, Art. 57 N23, mit Hinweisen auf die vom Bundesrat wiederholt vertretene a.A.; SGK BV-SCHWEIZER/MOHLER, Art. 57 N 4 f.; MOECKLI (Fn. 72), N9; MOHLER (Fn. 44), N37f.

80 Vgl. Botschaft PMT (Fn. 8), S. 4790 und 4801; AB2020 N 1126.

81 MOHLER, Verfassungsgrundlage (Fn. 44), N 54 ff., findet auch gesamtschweizerische Konkordate unbefriedigend und plädiert für eine Klärung der Zuständigkeiten im Rahmen einer Revision der BV.

82 BIAGGINI, OFK BV, Art.123 N4; SGK BV-VEST, Art. 123 N 2.

83 BGE137I 31 E. 4.3 und 4.4; BGE140 I2 E. 6.1; zustimmend BIAGGINI, OFK BV, Art. 123 N3. Vgl. auch LUBISHTANI/MONOD (Fn. 21), S. 24 f.; MOHLER, Verfassungsgrundlage (Fn. 44), N $43 \mathrm{ff}$.

84 BIAGgINI, OFK BV, Art. 173 N33 und Vorbem. Art. 42-135 N19; BSK BV-MERKER/CONRADIN, Art. 173 N134; SGK BV-SÄGESSER, Art. 173 N167 ff.; a.A. Botschaft PMT (Fn. 8), S. 4845 und 4782. 
existieren. ${ }^{85}$ So macht der Bundesrat gestützt auf BGE 117 Ia 202 eine inhärente Kompetenz im Bereich der inneren Sicherheit geltend, ${ }^{86}$ blendet aber einen wichtigen Teil des damaligen Leitentscheides aus. Das Bundesgericht wies nämlich darauf hin, dass der Bund die grundsätzliche verfassungsrechtliche Ordnung beachten müsse. Das gelte insbesondere für die Zuständigkeiten der Kantone, welchen «für ihr Gebiet primär die allgemeine Polizeihoheit» zukomme. ${ }^{87}$ Die inhärente Kompetenz des Bundes im Bereich der Gefahrenabwehr beschränkt sich auf den Schutz seiner eigenen Institutionen und Organe sowie auf die dem Bund völkerrechtlich obliegenden Schutzpflichten (insbesondere hinsichtlich des diplomatischen Personals und der internationalen Organisationen). ${ }^{88}$ Da nicht aus jedem Terroranschlag in einem Kanton eine solche Gefährdung des Bundes folgt, 89 kann daraus auch keine allgemeine Zuständigkeit dieser Staatsebene abgeleitet werden. ${ }^{90}$ Nicht überzeugend ist es schliesslich, aus einem - sachlich nachvollziehbaren - Koordinationsbedarf auf eine verfassungsrechtliche Kompetenz zu schliessen. ${ }^{91}$ Damit könnte der Bund faktisch nach Belieben in kantonale Zuständigkeiten eingreifen. ${ }^{92}$

Im Ergebnis stellen die im Ingress des PMT genannten Bestimmungen keine hinreichende Verfassungsgrundlage für die vorgesehene Teilrevision des BWIS dar. Es fehlt dem Bund an der Rechtsetzungskompetenz zum Erlass der polizeilichen «Massnahmen zur Verhinderung terroristischer Aktivitäten». Vielmehr wären die Kantone zuständig, welche über die originäre Polizeihoheit verfügen. ${ }^{93}$ Bei einem Inkrafttreten des PMT würde ihm dies aufgrund von Art.190 BV zwar formell kaum schaden, aber es ist doch sehr bedenklich, wenn der Bund in einem derart bedeutenden Bereich ohne verfassungsrechtliche Legitimität tätig wird. ${ }^{94}$

85 BIAGGINI, OFK BV, Art. 173 N 33; BSK BV-MEKER/CONRADIN, Art. 173 N 134; SGK BV-SÄGESSER, Art. 173 N 169.

86 Botschaft PMT (Fn. 8), S. 4845 und insb. dortige Fn. 114.

87 BGE117 Ia 202E. 5.

88 BIAGGINI, OFK BV, Art. 57 N6; BSK BV-DIGGELMANN/ALTWICKER, Art. 57 N 38, 46 und N27 ff.; MOECKLI (Fn. 72), N15 und 18; MOHLER, Verfassungsgrundlage (Fn. 44), N 5 ff. und 35f.;SGK BV-sCHWEIZER/ MOHLER, Art. 57 N6; vermittelnd TSCHANNEN, Normenarchitektur (Fn. 44), N13.

89 So wurde über die Attacken in Lugano und Morges 2020 überregional berichtet. Daraus eine Gefährdung des Bundes abzuleiten, würde aber zu weit gehen. Vgl. etwa FLORIAN SCHOOP, Der Staat muss auf diese Einzeltäter reagieren, NZZ vom 2. Dezember 2020, S.17.

90 Ausführlich MOHLER, Verfassungsgrundlage (Fn. 44), N25ff.

91 So aber Botschaft PMT (Fn. 8), S. 4763 f. und 4845 f.

92 LUBISHTANI/MONOD (Fn. 21), S. 25; MOECKLI, (Fn. 72), N9; a.A. TSCHANNEN, Normenarchitektur (Fn. 44), N15.

93 Gl.M. MOHLER, Verfassungsgrundlage (Fn. 44), N 47. Vgl. auch LUBISHTANI/MONOD (Fn. 21), S. 26; ZIMMERLIN (Fn. 5), S. 189.

94 Ausführlich MOHLER, Verfassungsgrundlage (Fn. 44), N46ff., m.w.H.

\section{Verfahrensrechtliche Stellung eines terroristischen Gefährders}

Soweit ergibt sich, dass die vielseitigen Massnahmen, 29 welche in das BWIS aufgenommen werden sollen, auf einer mangelhaften Grundlage beruhen. Gleichzeitig knüpfen sie an vagen Bestimmungen an, obwohl sie zu verschiedenen Grundrechtseinschränkungen führen können. ${ }^{95}$ Hinzu kommt, dass die Massnahmen verwaltungsrechtlicher Natur sind und die Vorschriften des Verwaltungsverfahrens zur Anwendung kommen. ${ }^{96} \mathrm{Da}-$ mit entgehen den betroffenen Personen die Verteidigungsrechte der StPO. ${ }^{97}$

Für die Zulässigkeit der geplanten Regelung spricht al- 30 lerdings, dass nach bundesgerichtlicher Rechtsprechung die Anforderungen an die Normdichte im Polizeirecht herabgesetzt sind. Dies hänge mit der Besonderheit des Regelungsbereichs zusammen. Die Aufgaben würden sich nur schwer abstrakt umschreiben lassen und die Arten und Formen der Gefährdung seien besonders vielgestaltig und wandelbar. ${ }^{98}$ Aus diesem Grund schützte das Bundesgericht auch schwerwiegende Grundrechtseinschränkungen, die gestützt auf eine vage formulierte Grundlage ergangen waren. ${ }^{99}$ Als Kompensation für mangelnde Normdichte verlangt das Bundesgericht aber verfahrensrechtliche Absicherungen zugunsten der Betroffenen. ${ }^{100}$ Es ist deshalb näher zu prüfen, wie die verfahrensrechtliche Stellung einer terroristischen Gefährderin zu beurteilen ist.

\section{Anordnung der Massnahmen}

Die Verfahrensherrschaft liegt klar beim fedpol: Es ver- 31 fügt die Massnahmen (nArt. 23j Abs. 1 BWIS), welche sofort in Kraft treten (nArt. $24 \mathrm{~g}$ Abs. 3 BWIS). ${ }^{101}$ Der Bundesrat erwog, dass dem fedpol damit der Entscheid über potenziell schwerwiegende Grundrechtseingriffe überantwortet wird-allerdings hat er sich explizit dagegen entschieden, diesen grundsätzlich von einer gerichtlichen Genehmigung abhängig zu machen. ${ }^{102}$ Dabei ist fedpol ein gewöhnliches Amt innerhalb des Eidgenössischen

\footnotetext{
95 Vgl. oben II.2.a) und CoNINX (Fn. 26), S. 12.

96 Vgl. oben II.2.b).

97 CONINX (Fn. 26), S. 12. LUBISHTANI/MONOD (Fn. 21), S. 23, sind dafür, mindestens auf die Eingrenzung auf eine Liegenschaft Art. 6 EMRK anzuwenden.

$98 \quad$ BGE136I87 E.3.1; BGE138I6 E.5.3.

99 REGINA KIENER / WALTER KÄLIN / JUDITH WYTTENBACH, Grundrechte, 3. Aufl., Bern 2018, §9 N79f., mit Hinweis auf BGE128I327.

100 BGE132I 49 E. 6.2; BGE136I 87 E. 3.1. Ebenso LUBISHTANI/MONOD (Fn. 21), S. 21, mit Hinweis auf BGE140 I381 E.4.4.

101 Vgl. oben II.2.c).

102 Botschaft PMT (Fn. 8), S. 4790. Ein anderslautender Minderheitsantrag wurde in der Parlamentsdebatte abgelehnt, vgl. AB 2020 N1127f.
} 
Justiz- und Polizeidepartements (Art. 9 ff. OV-EJPD ${ }^{103}$ ) und in die entsprechende Hierarchie eingebunden. ${ }^{104}$

Tätig wird fedpol auf Antrag von anderen Behörden, welche die relevanten Unterlagen beibringen (nArt. $23 \mathrm{i}$ BWIS). Auf die Anhörung der betroffenen Person vor Erlass der Verfügung soll hingegen regelmässig verzichtet werden, um der Gefährdung möglichst rasch zu begegnen. ${ }^{105}$ Hinzu kommen kann, dass in Akten (etwa des NDB) auch nachträglich keine Einsicht gewährt wird, weil sie als geheim eingestuft werden. ${ }^{106}$ Dies ist zulasten einer Partei zulässig, wenn ihr die Behörde vom wesentlichen Inhalt Kenntnis und die Gelegenheit gibt, sich dazu $\mathrm{zu}$ äussern und Gegenbeweise einzureichen (Art. 28 VwVG). ${ }^{107}$ Die genannten Einschränkungen stehen zumindest in einem Spannungsverhältnis mit dem Anspruch auf rechtliches Gehör i.S.v. Art. 29 Abs. 2 BV und Art. 30 Abs. 1 VwVG. ${ }^{108}$

Allerdings sieht Art. 30 Abs. 2 lit. e VwVG vor, dass auf eine vorgängige Anhörung der betroffenen Person verzichtet werden kann, wenn Gefahr im Verzug ist, d.h. überwiegende Interessen zu wahren sind. ${ }^{109}$ Vorliegend dürfte die Wahrung der öffentlichen Sicherheit und Ordnung im Zentrum stehen, was ein gewichtiges öffentliches Interesse darstellt.110 Nach höchstrichterlicher Praxis muss zur Berufung auf diesen Ausnahmetatbestand kumulativ zeitliche Dringlichkeit bestehen, die Parteien müssen eine Beschwerdeinstanz anrufen können, welche über volle Kognition verfügt, und es darf keine andere Bestimmung des Bundesrechts einen Anspruch auf vorgängige Anhörung gewährleisten. ${ }^{111}$ Der zu verhindernde Nachteil muss aufgrund objektiver Anhaltspunkte wahr-

103 Organisationsverordnung für das Eidgenössische Justiz- und Polizeidepartement vom 17. November 1999 (OV-EJPD; SR 172.213.1).

104 Vgl. zur Hierarchie in der Zentralverwaltung statt vieler: PIERRE TSCHANNEN / ULRICH ZIMMERLI / MARKUS MÜLLER, Allgemeines Verwaltungsrecht, 4. Aufl., Bern 2014, §6 N4ff.

105 Vgl. oben II.2.c), insb. ZIMMERLIN (Fn. 5), S. 197, wonach dies «taktisch geradezu geboten» sei.

106 ZIMMERLIN (Fn. 5), S. 197.

107 Vgl. STEPHAN C. BRUNNER, in: Auer/Müller/Schindler (Hrsg.), VwVG Kommentar, 2. Aufl., Zürich 2019, Art. 28 N1ff. (zit. Komm. VwVGBEARBEITERIN); BERNHARD WALDMANN / MAGNUS OESCHGER, in Waldmann/Weissenberger (Hrsg.), Praxiskommentar Verwaltungsverfahrensgesetz, 2. Aufl., Zürich 2016, Art. 28 N1ff. (zit. PraxKomm. VwVG-BEARBEITERIN).

108 KIENER/KÄLIN/WYTTENBACH (Fn. 99), §41 N 40 ff.; Komm. VwVGSUTTER, Art. 30 N1ff.; PraxKomm. VwVG-WALDMANN/BICKEL, Art. $30 \mathrm{~N} 15 \mathrm{ff}$.

109 KIENER/RÜTSCHE/KUHN (Fn. 65), N658f. und 663; PraxKomm. VwVG-WALDMANN/BICKEL, Art. $30 \mathrm{~N} 51$ und 84f.

110 Vgl. TIEFENTHAL (Fn.15), §5N16f.; TSCHANNEN, Staatsrecht(Fn.74), $\$ 1 \mathrm{~N} 10$.

111 BGE104 Ib 129 E. 3 und 4; BGE128V 272 E. 5.b/ee; zustimmend AL FRED KÖLZ / ISABELLE HÄNER / MARTIN BERTSCHI, Verwaltungsverfahren und Verwaltungsrechtspflege des Bundes, 3. Aufl., Zürich 2013, N532. scheinlich sein.112 Zudem gebietet das Verhältnismässigkeitsprinzip, dass der Gehörsanspruch so rasch und so weit als möglich nachgeholt wird. ${ }^{113}$ Der Ausnahmetatbestand ist restriktiv zu handhaben. Eine nachträgliche Anhörung ist meist nur ein unvollkommener Ersatz für die vorgängige Mitwirkung im Verfahren; insbesondere muss die Betroffene gegen eine bereits erlassene Verfügung vorgehen, was regelmässig einen gesteigerten Begründungsaufwand nach sich zieht. ${ }^{114}$

Grundsätzlich zulässig und üblich ist, dass eine Behörde 34 nur mittelbar Beweise abnimmt (vgl. Art.14 VwVG). ${ }^{115}$ Eine Ausnahme wird von der Lehre allerdings dort gefordert, wo es massgeblich auf die unmittelbare Wahrnehmung eines Beweismittels, wie etwa das nonverbale Verhalten eines Zeugen, ankommt. ${ }^{116}$ Dies würde in den hier interessierenden Konstellationen dafür sprechen, dass zumindest der Betroffene vor Erlass der Verfügung angehört wird.

Insgesamt ist festzuhalten, dass das Bundesverwaltungs- 35 gericht zwar über volle Kognition verfügt (Art. 49 VwVG i.V.m. Art. 31 VGG ${ }^{117}$ ) und keine Norm ersichtlich ist, welche eine vorgängige Anhörung der Betroffenen bei BWISMassnahmen verlangen würde. Allerdings ist mit Blick auf die Verhältnismässigkeit (Art. 5 Abs. 2 und Art. 36 Abs. $3 \mathrm{BV}$ ) mindestens fraglich, ob regelmässig ganz auf die vorgängige Gewährung des rechtlichen Gehörs verzichtet werden darf. Umso weniger ist dies m.E. angezeigt, je mehr dabei auf Akten abgestellt wird, welche auch nachträglich nicht offengelegt werden (Art. 28 VwVG). Ebenfalls heikel sind die Fälle, in welchen für mehrere Monate eine Eingrenzung auf eine Liegenschaft i.S.v. nArt. 230 BWIS verfügt und diese vollzogen wird, während der Betroffene den Rechtsmittelweg durchlaufen muss und die sozialen Folgen des Vollzugs zu tragen hat. ${ }^{118}$

Nicht vertieft wird hier die Konformität dieser Eingren- 36 zung mit Art. 5 EMRK und Art. 31 Abs. 4 BV. DONATSCH und CONINX haben dies eingehend dargestellt und verneinen eine Vereinbarkeit der vorgesehenen Regelung

\footnotetext{
112 PraxKomm. VwVG-wALDMANN/BICKEL, Art. 30 N72, m.w.H.

113 KIENER/KÄLIN/WYTTENBACH (Fn. 99), \$41 N 51; vgl. auch BGE138I6 E.6.3.

114 BGE126 II 111 E. 6b/aa; Komm. VwVG-SUTTER, Art. 30 N 30; PraxKomm. VwVG-WALDMANN/BICKEL, Art. $30 \mathrm{~N} 73$ und 84.

115 KIENER/RÜTSCHE/KUHN (Fn. 65), N693; KÖLZ/HÄNER/BERTSCHI (Fn.111), N250f.

116 PraxKomm. VwVG-wEISSENBERGER/HIRZEL, Art.14 N44f.; zustimmend KIENER/RÜTSCHE/KUHN (Fn. 65), N693.

117 Bundesgesetz über das Bundesverwaltungsgericht vom 17.Juni 2005 (VGG; SR 173.32).

118 Vgl. demgegenüber Art. 224f. StPO zur Untersuchungshaft, wonach die Beschuldigte jeweils «unverzüglich» durch die Staatsanwaltschaft und sodann durch das Zwangsmassnahmengericht zu hören ist.
} 
mit dem übergeordneten Recht. ${ }^{119}$ Hinsichtlich des Verfahrens sei aber Folgendes angemerkt: Wenn nArt. 23p Abs. 4 BWIS vorsieht, dass die betroffene Person ein Gesuch um Aufhebung der Massnahme zuerst beim fedpol einreichen muss, bevor innert spätestens acht Tagen ein Gerichtsentscheid vorliegt, so ist dies nicht in allen Fällen vereinbar mit dem Anspruch, «jederzeit ein Gericht anzurufen». Erstens sind acht Tage eine lange Dauer. ${ }^{120}$ Zweitens gelangt die Betroffene nicht direkt an ein Gericht, wie es grundrechtlich verlangt wäre, wenn der Freiheitsentzug nicht auf einem gerichtlichen Urteil beruht was in dem Zeitpunkt der Fall ist. ${ }^{121}$ Die Massnahme nach nArt. 230 BWIS kann während den ersten 48 Stunden allein auf einem Entscheid des fedpol basieren (nArt. 23p Abs.1BWIS). Bis zum Entscheid desZwangsmassnahmengerichts müsste es zulässig sein, direkt mit dem eigenen Standpunkt von diesem gehört zu werden. Das muss auch zeitlich möglich sein, wenn bedacht wird, dass etwa der Polizeigewahrsam i.S.v. \$27 Abs. 1 Satz1 PolG/ZH überhaupt nur für 24Stunden angeordnet werden darf, aber ab Beginn desselben eine gerichtliche Überprüfung verlangt werden kann (\$27 Abs. 1 Satz 2 PolG/ZH). ${ }^{122}$

\section{Beschwerde an das Bundesverwaltungsgericht}

37 Einer Beschwerde gegen die Anordnung von BWIS-Massnahmen kommt grundsätzlich keine aufschiebende Wirkung zu (nArt. 24g Abs. 3 BWIS). Dabei hat der Betroffene nicht nur die Schwierigkeit, dass er gegen eine sich bereits im Vollzugbefindliche Verfügung vorgehen muss. ${ }^{123}$ Es obliegt ihm auch der Nachweis, dass die Prognose über eine von ihm ausgehende Gefahr nicht zutrifft. Er müsste also etwa beweisen können, dass aus seinen Handlungen in den sozialen Medien nicht auf eine terroristische Aktivität geschlossen werden kann. ${ }^{124}$ Ein negativer Beweis, der sich wohl nur sehr schwer erbringen lässt, weshalb das Bundesgericht solche generell nur mit Zurückhaltung fordert. ${ }^{125}$ Das Bundesverwaltungsgericht begegnet der Schwierigkeit mit der Herabsetzung des Beweismasses und der Verpflichtung der Gegenpartei, substanziierte

119 CONINX (Fn. 26), S.13ff.; DONATSCH (Fn. 40), N31ff. und insb. N127ff. Vgl. auch ZIMMERLIN (Fn. 5), S.194f.

120 KIENER/KÄLIN/WYTTENBACH (Fn. 99), \$44 N36, geben als zulässige Grenze unter Berufung auf den UNO-Menschenrechtsausschuss eine Frist von bis zu sieben Tagen an. Zustimmend RHINOW et al. (Fn.67), N611.

121 BIAGGINI, OFK BV, Art. 31 N16; BSK BV-SCHÜRMANN, Art. 31 N $51 \mathrm{ff}$.; SGK BV-VEST, Art. 31 N 40 ff.; BGE136 I 87 E. 6.5.2.

122 Vgl. BEAT OPPLIGER / STEFAN HEIMGARTNER, in: Donatsch/Jaag/ Zimmerlin (Hrsg.), Kommentar zum Polizeigesetz des Kantons Zürich, Zürich 2018, \$27 N9ff.

123 Vgl. oben IV.1.

124 Vgl. oben II.2.a).

125 BGE137 II 313 E. 3.5.2, m.w.H. auf die Rechtsprechung. Vgl. auch PraxKomm. VwVG-KRAUSKOPF/EMMENEGGER/BABEY, Art.12 N 213.
Indizien für ihren Standpunkt zu benennen. ${ }^{126}$ Sollte das PMT in Kraft treten, wird zu prüfen sein, ob die vagen Anhaltspunkte, welche zur Annahme einer terroristischen Gefährdung genügen sollen, auch substanziierte Indizien im Sinne dieser Rechtsprechung darstellen.

Eine Inkonsistenz in der Gesetzgebung zeigt sich in dem 38 Fall, in welchem das Zwangsmassnahmengericht die Genehmigung zur Eingrenzung auf eine Liegenschaft verweigert oder einem Gesuch um Aufhebung der Massnahme entspricht. Nach nArt. 23p Abs. 5 lit. b und c BWIS hat fedpol diese sodann unverzüglich zu beenden. Wenn es die Auffassung des Gerichts nicht teilt - also von einer terroristischen Gefahr ausgeht - so steht ihm dagegen zwar die Beschwerde an das Bundesverwaltungsgericht zu, allerdings hat diese auch in dem Fall keine aufschiebende Wirkung (vgl. nArt.24g Abs. 2 lit.b und Abs. 3 BWIS). ${ }^{127}$ Die terroristische Gefährderin wäre also während dem laufenden Beschwerdeverfahren frei. Ein Ergebnis, welches sich nur schwer mit dem Sinn und Zweck des PMT vereinbaren lässt.

Hinzu kommt, dass die Konzentration des Rechtsmittel- 39 wegs beim Bundesverwaltungsgericht nur für die Massnahmen nach BWIS gilt. Da diese auch während einem Strafverfahren angeordnet werden können (nArt. $23 f$ Abs. 1 lit. c BWIS) und der Vollzug der Massnahmen durch die Kantone erfolgt (nArt. 23 r Abs. 1 BWIS), ${ }^{128}$ sind Konstellationen möglich, in welchen neben dem Bundesverwaltungsgericht auch die kantonale Beschwerdeinstanz in Strafsachen (Art. 393 ff. StPO) und die kantonale Beschwerdeinstanz im Verwaltungsverfahren mit der materiell selben Sache befasst werden müssen. ${ }^{129}$ Die PMTVorlage macht nicht klar, wie diese drei Gerichtsverfahren zu koordinieren sind. Aus Sicht der Betroffenen ist m.E. zu fordern, dass sämtliche Verfahren unter besonderer Berücksichtigung des Beschleunigungsgebots behandelt werden, um diese mehrfach belastende Situation nicht unnötig zu verlängern. Zudem sollten sich die entscheidenden Instanzen ins Vernehmen setzen, um sich widersprechende Urteile möglichst zu vermeiden.

Nicht dargestellt wird hier der sich anschliessende 40 Rechtsmittelweg an das Bundesgericht. Dieses ist grundsätzlich auf eine Rechtskontrolle beschränkt (Art. 95 BGG) und deshalb nur unvollkommen in der Lage, Mängel im vorhergehenden Verfahren zu heilen. ${ }^{130}$

126 Urteil des Bundesverwaltungsgerichts A-5113/2014 vom 11. Dezember 2014 E. 4.1.3, unter Hinweis auf BGE137 II 313 und BGE139 II 451 E. 2.4; Rechtsprechung bestätigt in Urteil des Bundesverwaltungsgerichts A-6931/2018 vom 20. September 2019 E. 2.2.

127 Vgl. oben II.2.c)

128 Vgl. oben II.2.c).

129 ZIMMERLIN (Fn. 5), S.197f.

130 Zu den zulässigen Beschwerdegründen vor Bundesgericht: KöLZ/ HÄNER/BERTSCHI (Fn. 111), N1541ff.; RHINOW et al. (Fn. 67), N1956ff. 


\section{Bewertung}

amt ist keine verfahrensrechtliche Kompensation zugunsten der von den BWIS-Massnahmen Betroffenen ersichtlich, obwohl dies aufgrund der mangelhaften Normdichte und Grundrechtsrelevanz der Bestimmungen notwendig wäre. Ihre Stellung ist gegenüber fedpol eher schwach ausgestaltet, was sich auch auf das Verfahren vor Bundesverwaltungsgericht auswirkt. Immerhin gelten dort gewisse Erleichterungen beim Erbringen eines negativen Beweises - dies stellt allerdings keine Privilegierung gegenüber anderen Rechtsmittelverfahren dar, die weniger grundrechtssensibel sind. Es bleibt damit der Befund, dass mit dem PMT erhebliche Grundrechtseinschränkungen aufgrund von Normen möglich sein sollen, die wenig präzis und klar sind, ohne dass diese mangelnde Normdichte gemäss den Anforderungen der Rechtsprechung kompensiert würde.

\section{Möglichkeiten einer alternativen Regelung}

Eine Verbesserung der jetzt vorliegenden Teilrevision des BWIS sollte m.E. die folgenden Punkte berücksichtigen: Der Anwendungsbereich in nArt. 23e BWIS ist enger zu fassen. Insbesondere sollte bei der Definition einer terroristischen Aktivität nicht auf eine blosse Androhung oder die Verbreitung von Furcht und Schrecken abgestellt werden. Ebenso ist die Umschreibung zu offen, wonach eine Begünstigung der Änderung der staatlichen Ordnung durch solche Handlungen schon ausreichend sein soll, um eine terroristische Aktivität zu bejahen. Darüber hinaus sollten sämtliche Massnahmen nur unter Vorbehalt der Zustimmung eines Gerichts angeordnet werden können. Das in nArt. 23p Abs.1 BWIS bereits erwähnte Zwangsmassnahmengericht des Kantons Bern wäre dazu sicher geeignet.

Hier nicht vertieft wurde die Vereinbarkeit der Eingrenzung auf eine Liegenschaft mit Art. 5 EMRK und Art. 31 Abs. 4 BV. Es ist aber nochmals hervorzuheben, dass neben CONINX auch DONATSCH, der Gutachter der kantonalen Justiz- und Polizeidirektorinnen und -direktoren in dieser Sache, die Vereinbarkeit der vorgesehenen Regelung mit dem übergeordneten Recht verneint hat. ${ }^{131}$ Auf diese Massnahme ist deshalb ganz zu verzichten.

131 CONINX (Fn. 26), S.13ff.; DONATSCH (Fn. 40), N31ff. und insb. N127ff.
Wenn die Altersgrenze nach nArt. 24f BWIS beibehalten 44 werden soll, so könnte in diese Norm immerhin ein dritter Absatz eingefügt werden, welcher die Behörden explizit zur Berücksichtigung einer allfälligen Minderjährigkeit verpflichtet. Verfahrensrechtlich sollte für unmündige Personen ein obligatorischer Rechtsbeistand und die Zuständigkeit eines Jugendgerichts (statt des Zwangsmassnahmengerichts) festgelegt werden.

Weiter sollte der Rechtsschutz bei einer einzigen gericht- 45 lichen Instanz konzentriert werden. Diese könnte zur möglichst weitgehenden Offenlegung der Akten und der Überprüfung der Angemessenheit verpflichtet werden. Dies tut nArt. 23p Abs. 1 BWIS bereits, wenn er das Zwangsmassnahmengericht «zur Prüfung der Rechtmässigkeit und Angemessenheit» anhält.

Schliesslich ist nochmals zu betonen, dass eigentlich nur 46 die Kantone kompetent sind, die hier betrachteten Massnahmen zu beschliessen. Denkbar wäre etwa eine interkantonale Vereinbarung in Entsprechung zum HooliganKonkordat.

\section{Fazit}

Zusammenfassend ergibt sich, dass der Kern des PMT， 47 die «Massnahmen zur Verhinderung terroristischer Aktivitäten», auf einer zumindest zweifelhaften verfassungsrechtlichen Grundlage beruhen. Vielmehr wären die Kantone zur entsprechenden Rechtsetzung kompetent. Es kommt hinzu, dass die geplanten Massnahmen einen weiten und wenig präzisen Anwendungsbereich haben, aber gleichzeitig präventive und erhebliche Grundrechtseingriffe ermöglichen würden. Diese Belastung der Betroffenen wird auch nicht durch ein entsprechendes Verfahren kompensiert. Im Gegenteil: Das Verfahren wird von fedpol - ein Amt in der Zentralverwaltung des Bundes - beherrscht und eine gerichtliche Prüfung findet grundsätzlich nur im Nachhinein und auf Begehren des Betroffenen statt.

Insgesamt weist das Gesetzespaket erhebliche Schwach- 48 punkte auf. Sollte es in der kommenden Volksabstimmung angenommen werden, wird es an Lehre und Praxis - vor allem an den Gerichten - sein, diese so gut als möglich zu beheben und an den Gesetzgeber zu appellieren, bald wieder eine Teilrevision des BWIS an die Hand zu nehmen. 